ISSN: 1808-8759 (cd-rom) 2359-6562 (on-line)

\title{
VINHOS DE MESA VARIETAIS DE UVAS AMERICANAS: ANÁLISES QUÍMICAS E ENERGÉTICA
}

\author{
Maíra Rodrigues Uliana', Waldemar Gastoni Venturini Filho², José Maria Oliveira ${ }^{3}$ \& José \\ Antonio Teixeira ${ }^{4}$
}

RESUMO: A indústria brasileira de vinhos tem uma característica notável que a distingui dos outros mercados. Enquanto o mercado estrangeiro só aceita produtos originários das variedades europeias (V. vinifera), no Brasil, produtos originários das variedades americanas (V. labrusca e V. bourquina) e híbridos também são aceitos. Vinhos varietais secos e suaves das variedades Bordô (seco e suave), Isabel (suave) e Máximo (seco), foram avaliados pelas seguintes determinações químicas: teor alcoólico; densidade; extrato seco total e reduzido; relação álcool em massa/extrato seco reduzido, açúcares redutores; acidez total, volátil e fixa; $\mathrm{pH}$; dióxido de enxofre total e livre; e valor energético. Todos os vinhos analisados apresentaram resultados dentro dos parâmetros estipulados pela legislação brasileira, fato positivo, uma vez que são comercializados. O vinho varietal Máximo apresentou um teor baixo de dióxido de enxofre livre e total, podendo acarretar problemas futuros com sua sanidade.

PALAVRAS-CHAVE: Bebida alcoólica; Bordô; Isabel; Máximo.

\section{Varietal Table Wines From American Grapes: Chemical And Energy Analisys}

\begin{abstract}
The Brazilian wine industry has a remarkable characteristic that distinguishes from other markets, while the foreign market only accepts products originating from European varieties (Vitis vinifera), in Brazil, products originating from American varieties (Vitis labrusca and Vitis bourquina) and hybrids are also accepted. Dry and sweet varietal wines from varieties Bordô (dry and sweet), Isabel (sweet) e Máximo (dry) were analyzed, by the following chemical standard analyses: alcohol content; density; total and reduced dry matter; alcohol/reduced dry extract ratio; reducing sugars; total, volatile and fixed acidity; $\mathrm{pH}$; total and free sulfur dioxide; and energy value. All analyzed wines presented results within the parameters set forth by Brazilian law, a positive fact, once they are commercialized. The varietal wine Máximo presented a low content of total and free sulfur dioxide, which may cause future problems with its sanity.
\end{abstract}

KEYWORDS: Alcoholic beverages; Bordô; Isabella; Máximo.

\section{INTRODUÇÃO}

O setor brasileiro de vinho apresenta uma característica marcante que o distingue de outros mercados; enquanto o mercado estrangeiro somente aceita produtos originários das variedades europeias ( $V$. vinifera), no Brasil, também são aceitos produtos originários das variedades americanas (V. labrusca e $V$. bourquina) e híbridos. Atualmente no país, cerca de $85 \%$ da produção de vinho é oriunda de variedades americanas e híbridos, enquanto que as variedades europeias representam cerca de $15 \%$ (FAO, 2013; UNIÃO BRASILEIRA DE VITIVINICULTURA, 2013).

\footnotetext{
1 Faculdade de Ciências Agronômicas-UNESP/Botucatu. E-mail: mruliana@gmail.com

2 Faculdade de Ciências Agronômicas-UNESP/Botucatu. E-mail: venturini@fca.unesp.br

${ }^{3}$ Centro de Engenharia Biológica - Universidade do Minho. E-mail: jmoliveira@deb.uminho.pt

${ }^{4}$ Centro de Engenharia Biológica - Universidade do Minho. E-mail: jateixeira@deb.uminho.pt
}

A legislação brasileira define vinho de mesa como aquele com teor alcoólico de 8,6 a $14 \%$ (em volume) e podendo conter até um atm de pressão a $20^{\circ} \mathrm{C}$; já vinho de mesa de uvas americanas como elaborado com uvas do grupo das uvas americanas e/ou híbridas, podendo conter vinhos de variedades Vitis vinifera (BRASIL, 2004).

As principais substâncias que constituem o vinho são: açúcares, alcoóis (etanol, principalmente), ácidos orgânicos, sais de ácidos minerais e orgânicos, compostos fenólicos, compostos nitrogenados, compostos voláteis e aromáticos (HASHIZUME, 1983).

O valor energético de um alimento pode ser determinado de três maneiras. Duas dessas são através do uso das tabelas de composição química. Onde, uma é feita por meio da formulação do produto (lista de ingredientes e quantidade dos mesmos, sendo necessária a composição química de cada ingrediente do alimento). A outra calcula diretamente o valor energético através da 
composição química do produto pronto, não sendo necessário a formulação deste produto; desta maneira, o valor energético de cada alimento é calculado a partir dos teores em proteínas, lipídios e glicídios, utilizando os coeficientes específicos que levam em consideração o calor de combustão e a digestibilidade. Cada componente tem seu valor energético determinado: 4 $\mathrm{kcal} \mathrm{g}^{-1}$ de proteína, $4 \mathrm{kcal} \mathrm{g}^{-1}$ de carboidrato e $9 \mathrm{kcal} \mathrm{g}^{-1}$ de gordura. A terceira maneira de se determinar o valor energético de um alimento é através da queima do mesmo em bomba calorimétrica, para a quantificação direta da energia bruta (MERRIL; WATT, 1973; SILVA; QUEIROZ, 2002; BRASIL, 2003; TABELA..., 2011).

As unidades utilizadas para quantificar energia dos alimentos e bebidas são o Joule (Sistema internacional) e a Quilocaloria. Quilocaloria (kcal) é a quantidade de calor necessária para aquecer um grama de água, de $1{ }^{\circ} \mathrm{C}$ (ou mais precisamente de $14,5^{\circ} \mathrm{C}$ para $15,5^{\circ} \mathrm{C}$ ); e Joule (J) é a energia (trabalho) decorrente da aplicação de uma força de 1 Newton (N) em uma distância de 1 metro, na direção de aplicação de tal força. Uma Quilocaloria corresponde a 4,1868 J. (ROZENBERG, 2002; FELTRE, 2004; TABELA..., 2011).

Os vinhos comuns têm grande aceitação pela população brasileira e representam a maior parte da produção, porém, não recebem muita atenção da comunidade científica, pois quase a totalidade das produções bibliográficas e artigos científicos são voltados para a produção dos vinhos finos. Em função deste fato este trabalho se justifica.

O objetivo deste trabalho foi caracterizar vinhos de mesa varietais produzidos com uvas americanas e/ou híbridos, através de análises químicas e energética.

\section{MATERIAL E MÉTODOS}

Foram utilizados os seguintes vinhos de mesa de uvas americanas e/ou híbridos:

$$
\begin{aligned}
& \checkmark \quad \text { vinho suave produzido com a } \\
& \text { variedade Isabel (ISu); } \\
& \checkmark \quad \text { vinho suave produzido com a } \\
& \text { variedade Bordô (BSu); } \\
& \checkmark \quad \text { vinho seco produzido com a variedade } \\
& \text { Bordô (BSe); } \\
& \checkmark \quad \text { vinho seco produzido com a variedade } \\
& \text { Máximo (MSe). }
\end{aligned}
$$

Os vinhos varietais Isabel e Bordô foram fornecidos por vinícolas localizadas na Serra Gaúcha, cidade de Caxias do Sul, Rio Grande do Sul (latitude 29¹0’00" Sul, longitude $51^{\circ} 10^{\prime} 40^{\prime \prime}$ Oeste); já os vinhos da variedade Máximo foram fornecidos por uma vinícola artesanal localizada no centro-oeste do estado de São Paulo, cidade de Lençóis Paulista (latitude 22³6’18" Sul, longitude $48^{\circ} 48^{\prime} 15^{\prime \prime}$ Oeste) (Figura 1), todos da safra 2008.

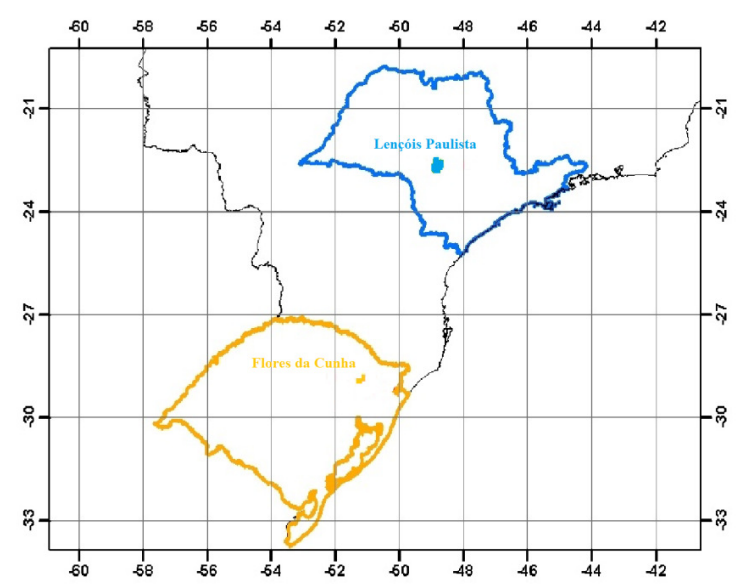

Figura 1 - Posição geográfica da origem dos vinhos.

\section{Análises realizadas:}

$\underline{\text { Teor alcoólico (TA) }}$

A determinação do TA foi realizada através da espectrometria de infravermelho próximo, método MI 108 (COMISSÃO DE VITICULTURA DA REGIÃO DOS VINHOS VERDES, 2011).

\section{Densidade}

Os vinhos foram submetidos a densimetria eletrônica, método MI 109 (COMISSÃO DE VITICULTURA DA REGIÃO DOS VINHOS VERDES, 2011), para determinação de suas densidades.

\section{$\underline{\text { Extrato seco total (AST) e reduzido (ESR) }}$}

Para a determinação do extrato seco total foi realizado cálculo seguindo o método OIV-MA-AS2-03B (ORGANISATION INTERNATIONALE DE LA VIGNE ET DU VIN, 2009). Já para o extrato seco reduzido foi feito o cálculo com base nos ensaios de açúcares totais e extrato seco total, de acordo com método OIV-MA-AS2-03B (ORGANISATION INTERNATIONALE DE LA VIGNE ET DU VIN, 2009).

\section{$\underline{\text { Relação álcool em massa/extrato seco reduzido (A/ESR) }}$}

Para a relação álcool (em peso)/extrato seco reduzido foi realizado cálculo de acordo com Brasil (2005).

Açúcares redutores (AR)

Para os vinhos secos: análise em fluxo segmentado, método MI 017 (COMISSÃO DE VITICULTURA DA REGIÃO DOS VINHOS VERDES, 2011); para os vinhos suaves: método de Luff-Schoorl, método MI 110 (COMISSÃO DE VITICULTURA DA REGIÃO DOS VINHOS VERDES, 2011). 


\section{Acidez total (AT), volátil (AV) e fixa (AF)}

Para a determinação da AT foi realizada a titulação potenciométrica, de acordo com o método OIV-MAAS313-01 (ORGANISATION INTERNATIONALE DE LA VIGNE ET DU VIN, 2009).

A AV foi efetuada através da destilação por arraste de vapor e titulação sem dosagem de interferentes, método MI 009 OIV-MA-AS313-02 (ORGANISATION INTERNATIONALE DE LA VIGNE ET DU VIN, 2009).

A AF foi feita diretamente por cálculo, de acordo com o método OIV-MA-AS313-03 (ORGANISATION INTERNATIONALE DE LA VIGNE ET DU VIN, 2009).

$\underline{\mathrm{pH}}$

De acordo com o método OIV-MA-AS313-15 (ORGANISATION INTERNATIONALE DE LA VIGNE ET DU VIN, 2009), por potenciometria.

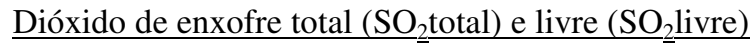
Para ambas análises foi utilizada a titulação iodométrica sem correção, seguindo o método MI 104 (COMISSÃO DE VITICULTURA DA REGIÃO DOS VINHOS VERDES, 2011).

Valor energético

A determinação energética dos vinhos foi realizada de forma direta, em bomba calorimétrica (Oxygen Combustion Bombda marca Parr, modelo 1108), de acordo com Silva e Queiroz (2002).

\section{Planejamento experimental e análise estatística}

Foram adquiridas 16 garrafas de vinho de $1 \mathrm{~L}$ (Bordô seco e suave e Isabel suave) e 0,75 L (Máximo seco) de um mesmo lote industrial (4 para cada variedade). $\mathrm{O}$ delineamento experimental foi o inteiramente casualizado, com quatro tratamentos e duas repetições. Foi realizada análise de variância e as médias foram comparadas pelo Teste Tukey $(\mathrm{p}<0,01)$, utilizando o software ASSISTAT, versão 7.6 beta (SILVA, 2012).

\section{RESULTADOS E DISCUSSÕES}

Nas quatro amostras, para todas as determinações, houve diferenças estatísticas significativas (Tabela 1).

Apesar dos vinhos BSe e BSu serem da mesma variedade, eles resultaram em TAs (teores alcoólicos) diferentes. Isto pode ser explicado pelos diferentes métodos de processamento dos vinhos, assim como pelas diferenças de locais e métodos de cultivos. Todos os vinhos apresentaram TAs dentro dos padrões estipulados pela legislação brasileira para vinhos de mesa (8,6 a 14 $\%$ em volume) (BRASIL, 2004) (Tabela 1).

As densidades dos vinhos acompanharam os ESTs (extratos secos totais). Em função da presença de açúcares, os vinhos suaves apresentaram densidades acima da unidade (Tabela 1). O TA também influenciou a densidade dos vinhos; o tratamento com maior teor alcoólico (MSe) apresentou a menor densidade. Isso se explica pela baixa densidade do etanol $\left(0,79 \mathrm{~g} / \mathrm{cm}^{3}\right)$.

Os resultados de densidade (Tabela 1) correspondem àqueles geralmente encontrados nesse tipo de vinho (RIZZON; MIELE; MENEGUZZO, 2000; RIZZON; MIELE, 2006; TECCHIO; MIELE; RIZZON, 2007).

De acordo com Sousa (2000) e Garruti (2001), o extrato seco representa os sólidos solúveis não voláteis que restam no vinho após a desidratação da amostra. Os vinhos suaves deste estudo, por conterem uma quantidade maior de açúcar, possuem maior EST; já os vinhos secos possuem uma pequena concentração de açúcar e consequentemente menor EST (Tabela 1).

Tabela 1 - Teor alcoólico, densidade, extrato seco total, extrato seco reduzido, relação álcool (em massa)/extrato seco reduzido e açúcares redutores de vinhos das variedades Isabel, Bordô e Máximo.

\section{Determinações}

\begin{tabular}{|c|c|c|c|c|c|c|}
\hline Amostras & $\begin{array}{l}\text { TA } \\
\text { (\% vol.) }\end{array}$ & $\begin{array}{l}\text { Densidade } \\
\left(\mathrm{g} \mathrm{mL}^{-1}\right)\end{array}$ & $\begin{array}{l}\text { EST } \\
\left(\mathrm{g} \mathrm{L}^{-1}\right)\end{array}$ & $\begin{array}{l}\text { ESR } \\
\left(\mathrm{g} \mathrm{L}^{-1}\right)\end{array}$ & $\begin{array}{l}\text { Relação } \\
\text { A/ESR }\end{array}$ & $\begin{array}{l}A R \\
\left(g \mathbf{L}^{-1}\right)\end{array}$ \\
\hline $\mathbf{I S u}$ & 10,1 b & $1,0399 \mathrm{~b}$ & 143,9 b & $20,8 d$ & 3,88 b & 123,1 a \\
\hline Bsu & 9,1 c & 1,0417 a & 145,5 a & 26,1 a & 2,79 d & 119,4 b \\
\hline BSe & 10,1 b & 0,9957 c & 28,4 c & 25,2 b & 3,21 c & $3,2 \mathrm{c}$ \\
\hline MSe & $12,7 \mathbf{a}$ & $\mathbf{0 , 9 9 1 7} \mathrm{d}$ & $26,2 \mathrm{~d}$ & 24,9 c & $4,09 \mathrm{a}$ & $1,3 \mathrm{~d}$ \\
\hline
\end{tabular}

Médias seguidas pela mesma letra não diferem estatisticamente entre si pelo Teste Tukey ao nível de $1 \%$ de probabilidade.

Legenda = TA: teor alcoólico; EST: extrato seco total; ESR: extrato seco reduzido; A/ESR: relação álcool em massa/extrato seco reduzido; AR: açúcares redutores; ISu: vinho suave varietal Isabel; BSu: vinho suave varietal Bordô; BSe: vinho seco varietal Bordô; MSe: vinho seco varietal Máximo. 
O ESR (extrato seco reduzido) é obtido pela diferença entre o extrato seco total e o teor de açúcar redutor do vinho, e representa o corpo do vinho (SOUSA, 2000; GARRUTI, 2001). O ESR dos vinhos estiveram entre 20,8 a 26,1 g L-1 (Tabela 1) e estão próximos aos encontrados na literatura para esses tipos de vinhos (RIZZON; MIELE; MENEGUZZO, 2000; MALGARIM, 2006; TECCHIO; MIELE; RIZZON, 2007).

Os varietais Bordô (BSu e BSe) apresentaram maior ESR (Tabela 1). Isto pode ser explicado, pois vinhos obtidos a partir dessas uvas apresentam grande quantidade de matéria corante (MAIA; CAMARGO, 2005).

Os vinhos com teores de extrato seco reduzido menores que $20 \mathrm{~g} \mathrm{~L}-1$ apresentam-se como leve ao paladar, enquanto os vinhos com $30 \mathrm{~g}$ L-1 ou mais são percebidos como encorpados (ZOECKLEIN et al., 1994). Portanto, todos os vinhos varietais desse estudo não podem ser considerados encorpados.

A relação A/ESR (relação álcool em massa/extrato seco reduzido) de todos os vinhos (Tabela 1) está dentro dos padrões de identidade e qualidade estipulados pela legislação brasileira (4,8 para vinhos tintos e 6,0 para vinhos rosados) (BRASIL, 1988) e também de acordo com o encontrado na literatura (RIZZON; MIELE; MENEGUZZO, 2000; RIZZON; MIELE, 2006; TECCHIO; MIELE; RIZZON, 2007).
Os teores de açúcares dos vinhos BSe e MSe (Tabela 1) são aqueles encontrados em vinhos secos, segundo legislação brasileira (até 5,0 g L-1) (BRASIL, 1991), sendo resultantes dos açúcares residuais da fermentação.

Já os teores de açúcares dos vinhos BSu e ISu são resultantes da adição de açúcares (normalmente na forma de sacarose) para que o vinho se torne doce, classificando-os, segundo legislação brasileira, como vinhos suaves (mais de $20 \mathrm{~g} \mathrm{L-1)}$ (BRASIL, 1991). Porém, os teores de açúcares analisados para esses vinhos foram bem elevados e isto pode ter sido realizado uma vez que o açúcar é utilizado para mascarar defeitos sensoriais de bebidas.

A acidez no vinho é normalmente dividida em duas categorias: fixa e volátil, sendo a acidez total resultado da combinação destas categorias. A AF (acidez fixa) dos vinhos é representada pelos ácidos tartárico, málico, láctico, succínico e cítrico (JACKSON, 2008). A AV (acidez volátil) é formada pelos ácidos voláteis, principalmente ácido acético, além de outros ácidos em menores teores, como o fórmico, butírico e propiônico (AMERINE; BERG; CRUESS, 1967).

A AT (acidez total) de todos os vinhos (Tabela 2) está dentro dos padrões de identidade e qualidade para vinhos de mesa (de 55 a 130 meq L-1) (BRASIL, 1988) e também condiz com os resultados encontrados na literatura (RIZZON; MIELE; MENEGUZZO, 2000; RIZZON; MIELE, 2006; TECCHIO; MIELE; RIZZON, 2007).

Tabela 2 - Acidez total, volátil e fixa, pH, dióxido de enxofre livre e total, e energia de vinhos das variedades Isabel, Bordô e Máximo.

\section{Determinações}

\begin{tabular}{|c|c|c|c|c|c|c|c|}
\hline Amostras & $\begin{array}{l}\mathrm{AT} \\
\left(\mathrm{meq} \mathbf{L}^{-1}\right)\end{array}$ & $\begin{array}{l}\mathrm{AV} \\
\left(\operatorname{meq} \mathbf{L}^{-1}\right)\end{array}$ & $\begin{array}{l}\text { AF } \\
\left(\operatorname{meq~L}{ }^{-1}\right)\end{array}$ & pH & $\begin{array}{l}\text { SO } O_{2} \text { livre } \\
\left(\mathrm{mg} \mathrm{L}^{-1}\right)\end{array}$ & $\begin{array}{l}\mathrm{SO}_{2} \text { total } \\
\left(\mathrm{mg} \mathrm{L}^{-1}\right)\end{array}$ & $\begin{array}{l}\text { Energia } \\
\left(\text { Kcal 100g }^{-1}\right)\end{array}$ \\
\hline ISu & 85,3 a & $5,07 \quad d$ & 78,7 a & 3,3 c & $26 \mathrm{c}$ & 198 a & 133,32 a \\
\hline Bsu & 76,0 b & 7,07 b & $66,7 \mathrm{~b}$ & 3,5 b & 39 b & $105 \mathrm{~b}$ & 132,08 a \\
\hline BSe & $72,0 \quad c$ & $6,14 \mathrm{c}$ & $64,0 \quad c$ & 3,5 b & $60 \mathrm{a}$ & $107 \mathrm{~b}$ & 112,97 b \\
\hline MSe & 72,0 c & 9,01 a & $61,3 \mathrm{~d}$ & $3,6 \mathrm{a}$ & $6 \mathrm{~d}$ & $21 \mathrm{c}$ & $109,95 \mathrm{~b}$ \\
\hline
\end{tabular}

Médias seguidas pela mesma letra não diferem estatisticamente entre si pelo Teste Tukey ao nível de $1 \%$ de probabilidade.

Legenda = AT: acidez total; AV: acidez volátil; AF: acidez fixa; SO2livre: dióxido de enxofre livre; SO2total: dióxido de enxofre total; ISu: vinho suave varietal Isabel; BSu: vinho suave varietal Bordô; BSe: vinho seco varietal Bordô; MSe: vinho seco varietal Máximo.

A baixa concentração da AV (menor que 13,3 meq $\mathrm{L}^{-1}$ ) indica a sanidade do vinho (AMERINE; BERG; CRUESS, 1967; ZOECKLEIN et al., 1994). Neste estudo, o teor da acidez volátil foi baixo em todos os casos (Tabela 2), indicando que os vinhos encontram-se em um estado sanitário favorável e estão dentro dos padrões de identidade e qualidade para vinhos de mesa (20 meq L ${ }^{-1}$ máximo) (BRASIL, 1988).

$\mathrm{O}$ pH dos vinhos é importante pelo efeito na cor, no sabor e relação do dióxido de enxofre livre e combinado. Para que o vinho atinja níveis satisfatórios dessas características o $\mathrm{pH}$ deve estar entre 3,1 e 3,6 
(AMERINE; BERG; CRUESS, 1967), o que ocorreu em todos os vinhos desse estudo (Tabela 2).

Com relação aos resultados de dióxido de enxofre total, todos os vinhos apresentaram teores dentro dos valores exigidos pela legislação (350 $\mathrm{mg} \mathrm{L}^{-1}$ máximo) (BRASIL, 1988). Porém, no vinho MSe, foi detectado teor de dióxido de enxofre (livre e total) muito baixo e, associado a um teor de acidez volátil mais alto; esse resultado nos indica que o vinho poderá sofrer alterações e contaminações e assim agravar seu estado de sanidade (Tabela 2).

Com relação aos resultados da determinação de valor energético, pode-se observar que os vinhos suaves (ISu e $\mathrm{BSu}$ ) resultaram em valores energéticos mais altos que os vinhos secos (BSe e MSe), decorrentes da presença de açúcares nesses vinhos (Tabela 1 e 2).

\section{CONCLUSÃO}

Nas condições experimentais empregadas, pode-se concluir que todos os vinhos analisados apresentaram-se dentro dos parâmetros estipulados pela legislação brasileira, fato positivo, uma vez que esses são comercializados no mercado brasileiro de bebidas.

Os teores de extrato seco e açúcar foram os componentes que mais contribuíram para o valor energético dos vinhos.

\section{REFFERENCIAS}

AMERINE, M. A.; BERG, H. W.; CRUESS, W. V. The technology of wine making. 2. ed. Westport: AVI, 1967. $797 \mathrm{p}$.

BRASIL. Decreto $n^{\circ} 113$, de 06 de maio de 1991. Altera decreto $\mathrm{n}^{\circ}$ 99066, de 8 de março de 1990, que regulamenta a Lei $\mathrm{n}^{\mathrm{o}} 7.678$, de 08 de novembro de 1988 . Diário Oficial da União, Brasília, DF, v. 1, n. 86, p. 8497-8498, 07 mai. 1991. Disponível em: $<$ http://sistemasweb.agricultura.gov.br/sislegis/action/det alhaAto.do?method=gravarAtoPDF\&tipo=DEC\&numer oAto $=00000113 \&$ seqAto $=000 \&$ valorAno $=1991 \&$ orgao $=$ NI $\& \operatorname{codTipo}=\&$ desItem $=\&$ desItemFim $=>$. Acesso em: 12 mar. 2013.

BRASIL. Lei ${ }^{\circ} 10.970$, de 12 de novembro de 2004. Altera dispositivos da Lei $\mathrm{n}^{\circ} 7.678$, de 8 de novembro de 1988, que dispõe sobre a produção, circulação e comercialização do vinho e derivados da uva e do vinho, e dá outras providências. Diário Oficial da União, Brasília, DF, v. 1, n. 219, p. 1-2, 16 nov. 2004. Disponível em: $<$ http://sistemasweb.agricultura.gov.br/sislegis/action/det alhaAto.do?method=gravarAtoPDF\&tipo=LEI\&numero Ato $=00010970 \&$ seqAto $=000 \&$ valorAno $=2004 \&$ orgao $=$ NI\&codTipo $=\& d$ esItem $=\&$ desItemFim $=>$. Acesso em: 12 mar. 2013.
BRASIL. Ministério da Agricultura. Portaria $\mathrm{n}^{\circ}$ 229, de 25 de outubro de 1988. Aprova as Normas referentes a "Complementação dos Padrões de Identidade e Qualidade do Vinho". Diário Oficial da União, Brasília, DF, v. 1, p. 20498, 31 out. 1988. Disponível em:

$<$ http://sistemasweb.agricultura.gov.br/sislegis/action/det alhaAto.do?method=gravarAtoPDF\&tipo=POR\&numer oAto $=00000229 \&$ seqAto $=000 \&$ valorAno $=1988 \&$ orgao $=$ MAPA\&codTipo $=\&$ desItem $=\&$ desItemFim $=>$. Acesso em: 12 mar. 2013.

BRASIL. Ministério da Saúde. Agência Nacional de Vigilância Sanitária. Resolução RDC n 360, de 23 de dezembro de 2003. Regulamento Técnico sobre Rotulagem Nutricional de Alimentos Embalados. Diário Oficial da União, Brasília, DF, v. 1, n. 251, p. 33-35, 26 dez. 2003. Disponível em:

$<\mathrm{http}$ ///sistemasweb.agricultura.gov.br/sislegis/action/det alhaAto.do?method=gravarAtoPDF\&tipo=RES\&numero Ato $=00000360 \&$ seqA to $=000 \&$ valorAno $=2003 \&$ orgao $=$ RDC/DC/ANVISA/MS\&codTipo $=\&$ desItem $=\&$ desItem Fim=>. Acesso em: 12 mar. 2013.

\section{COMISSÃO DE VITICULTURA DA REGIÃO DOS VINHOS VERDES. Anexo Técnico de Acreditação $\mathbf{N}^{\mathbf{0}}$ L0226-1. 17 ed. Porto, 2011. 6 p.}

FAO. Faostat agriculture data - crops production, crops processed production and food supply - grape and wine. 2013. Disponível em: <http://faostat.fao.org/?lang=en>. Acesso em: 11 mar. 2013.

FELTRE, R. Química: físico-química. 6. ed. São Paulo: Editora Moderna, 2004. v. 2, 417 p.

GARRUTI, D. S. Composição de voláteis e qualidade de aroma do vinho de caju. 2001. 200 p. Tese (Doutorado em Ciência dos Alimentos) - Faculdade de Engenharia de Alimentos, Universidade Estadual de Campinas, Campinas, 2001.

HASHIZUME, T. Fundamentos de tecnologia do vinho. In: AQUARONE, E.; LIMA, U. de A.; BORZANI, W. (Coord.) Alimentos e bebidas produzidos por fermentação. São Paulo: Blücher, 1983. p. 14-43.

JACKSON, R. S. Wine science: principles and applications. 3 ed. San Diego: Academic Press, 2008. $771 \mathrm{p}$.

MAIA, J. D. G.; CAMARGO, U. A. Sistema de produção de uvas rústicas para processamento em regiões tropicais do brasil. Bento Gonçalves: EMBRAPA Uva e Vinho, 2005. Disponível em: $<$ http://sistemasdeproducao.cnptia.embrapa.br/FontesHT ML/Uva/UvasRusticasParaProcessamento/cultivares.htm >. Acesso em: 27 out. 2009.

MALGARIM, M. B.; TIBOLA, C. S.; FERRI, V. C.; ZAIKOVSKI, C. B.; MANFROI, V. Características de qualidade do vinho 'Bordô' elaborado com diferentes 
processos de vinificação e períodos de maceração. Acta

Scientiarum Technology, Maringá, v. 28, n. 2, p. 199-

204, 2006.

MERRIL, A. L.; WATT, B. K. Energy value of foods: basis and derivation, revised. (Agricultural Handbook n. 74). Washington, DC: USDA, 1973. 105 p.

ORGANISATION INTERNATIONALE DE LA VIGNE ET DU VIN.Compendium of international methods of wine and must. Paris, 2009. v. 1.

Disponível em: <http://news.reseauconcept.net/images/oiv_es/Client/Compendium_2009_V ol1_EN.pdf>. Acesso em: 18 jan. 2011.

RIZZON, L. A.; MIELE, A. Efeito da safra vitícola na composição da uva, do mosto e do vinho Isabel da Serra Gaúcha, Brasil. Ciência Rural, Santa Maria, v. 36, n. 3, p. 959-964, 2006.

RIZZON, L. A.; MIELE, A.; MENEGUZZO, J.

Avaliação da uva cv. Isabel para a elaboração de vinho tinto. Ciência e Tecnologia de Alimentos, Campinas, v. 20, n. 1, p. 115-121, 2000.

ROZENBERG, I. M. Química geral. 1. ed. São Paulo: Blücher, 2002. 676 p.

SILVA, D. J.; QUEIROZ, A. C. Análises de alimentos: métodos químicos e biológicos. 3. ed. Viçosa:

Universidade Federal de Viçosa, 2002. 235 p.

SILVA, F. de A. S. Assistência estatística por Prof.

Dr. Francisco de Assis Santos e Silva: assistat versão 7.6 beta. Campina Grande: DEAG-CTRN-UFCG, 2012.

SOUSA, S. I. de. Vinho: aprenda a degustar. 1. ed. São Paulo: Market Press, 2000. 284 p.

TACO. Tabela brasileira de composição de alimentos. 4. ed. Campinas: NEPA (Núcleo de Estudos e Pesquisas em Alimentação) - UNICAMP, 2011. 161 p.

TECCHIO, F. M.; MIELE, A.; RIZZON, L. A.

Composição físico-química do vinho Bordô de Flores da Cunha, RS, elaborados com uvas maturadas em condições de baixa precipitação. Ciência Rural, Santa Maria, v. 37, n. 5, p. 1480-1483, 2007.

\section{UNIÃO BRASILEIRA DE VITIVINICULTURA.}

Dados estatísticos. Bento Gonçalves, 2012. Disponível em: <http://www.uvibra.com.br/pdf/safra_uva20022011.pdf>. Acesso em: 11 mar. 2013.

ZOECKLEIN, B. W.; FUGELSANG, K. C.; GUMP, B. H.; NURY, F. S. Wine analysis and production. New York: Chapman \& Hall, 1994. 621 p. 